\title{
Modelica Modeling and Simulation for a Micro Gas-Cooled
}

\section{Reactor}

\author{
Huimin Zhang ${ }^{1}$ Erhui Chen ${ }^{1}$ Yangyang Liang $^{1}$ Li Wang $^{1}$ Jun Wang ${ }^{1}$ Shuhong Du ${ }^{1}$ \\ Liping Chen ${ }^{2}$ Fanli Zhou ${ }^{2}$ Ji Ding ${ }^{2}$ Haiming Zhang ${ }^{2}$ \\ ${ }^{1}$ China Nuclear Power Engineering Co., Ltd., Beijing, China, \\ \{zhanghm, cheneh, liangyya, wanglie, wangjuna, dush\}@enpe.cc \\ ${ }^{2}$ Suzhou Tongyuan Software \& Control Technology Co., Ltd. Suzhou, China, \{chenlp, \\ zhoufl, dingj\} zhanghm\} @tongyuan.cc
}

\begin{abstract}
Highly compact micro nuclear reactors which have broad energy advantages in the application of ocean, land, space, and sky, become a hot research topic in the international nuclear industry recently. In this paper, Modelica language was used in the system modeling and simulation of a micro gas-cooled reactor. The Modelica model was self-developed by China Nuclear Power Engineering Company and the MWorks developed by Suzhou Tongyuan was chosen as the simulation platform. Two simulations of a concept micro gas-cooled reactor design were carried out. One is the extreme accident scenario and the other is a normal load-following operation. The simulation results showed that the reactor has good inherent safety even under the extreme accident, in which the reactor shutdown can be achieved only by the negative reactivity result from the increase of core temperature and the fuels were not damaged since the decay heat was removed by passive air cooling from outside of the reactor pressure vessel. The reactor also has good load-following performance, which can be achieved by simply adjusting the helium inventory (or pressure) and the control rod position, while the core temperature and power generation efficiency kept constant.
\end{abstract}

Keywords: system modeling, system simulation, micro-nuclear reactor, load-following

\section{Introduction}

The advanced micro nuclear energy system is a specific nuclear energy technology with high flexibility and sustainable and reliable energy supply, and has a good application prospect in remote areas, islands and other places with poor traffic and difficult energy supply. The micro nuclear energy system with the corresponding facilities can achieve the stable supply of clean energy, and has broad energy advantages in the application of ocean, land, space, and sky.

The concept micro gas-cooled reactor researched in this paper is a typical micro nuclear energy system with complex structures and multi-disciplinary, such as neutron physics, thermal engineering, energy conversion, electricity and control. Compared with a large-scale nuclear plant which adopt the traditional 'divide and conquer' design concept and is composed of a large amount of fully decoupled subsystems, the compact micro nuclear reactor has fewer subsystems but its subsystems are tightly coupled due to the constraints of the volume and weight. To handle the complexity aroused from this coupling issue and to better predict the reactor dynamic behavior, it is necessary to perform the system simulation across multiple disciplines and domains. Modelica is a unified modeling language for complex physics systems with multi-disciplinary. Oak Ridge National Laboratory (ORNL) adopted Dymola platform based on Modelica to establish the reactor model library TRANSFORM for the system modeling and simulation of high temperature gas-cooled reactor (HTGR) (Hale et al. 2015), nuclear thermal propulsion rocket(Rader et al. 2019)and molten salt reactor(Greenwood 2018; Greenwood et al. 2018). In China, MWorks platform based on Modelica developed by Suzhou Tongyuan was used for the integrated system simulation of manned spacecraft (Bainan et al. 2020) and two-phase flow (Yanping et al. 2021). In this paper, the MWorks platform was used for the system modeling and simulation of the concept micro gas-cooled reactor.

The whole system of the micro gas-cooled reactor is shown in Figure 1. The direct Brayton cycle is used for the heat-work conversion. As the medium of heat transfer and work, the helium is heated by the reactor core and then enters the turbine to expand, and then enters the recuperator to reduce the temperature. After further cooling by the precooler, the helium flows across the two-stage compressor to increase the pressure. Finally, the helium is heated by the recuperator and returns to the reactor core to repeat 
the thermal cycle process. The compressors, turbine and generator are connected by a main shaft, and the turbine provides torque power to drive the generator and compressors to rotate.

As shown in Figure 2, the reactor core is composed of 36 fuel bricks, an inner reflector assembly, an outer reflector, a core barrel and 12 control drums. Graphite is used as both the neutron moderator and the fuel structure, and is the major material of the fuel bricks, reflectors and control drums. The coated fuel particles TRISO (the design temperature limit is $1600^{\circ} \mathrm{C}$ (Yang et al. 2010)) are dispersed in the fuel bricks. The coolant channels are distributed regularly inside the fuel blocks as holes.

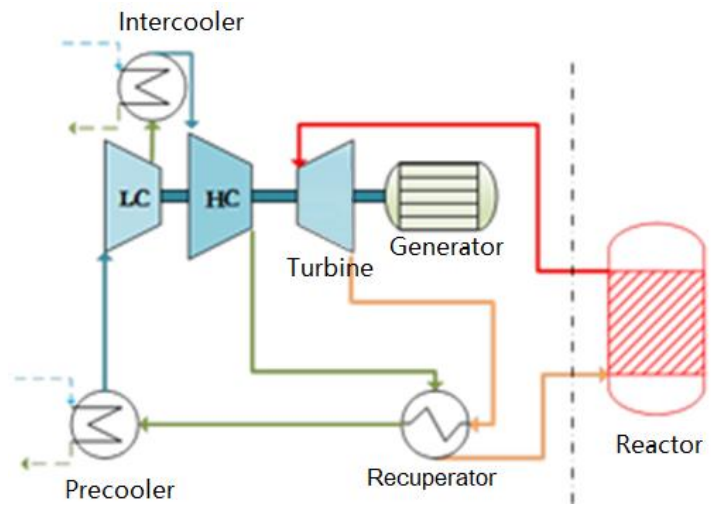

Figure 1. Main system of the micro gas-cooled reactor.

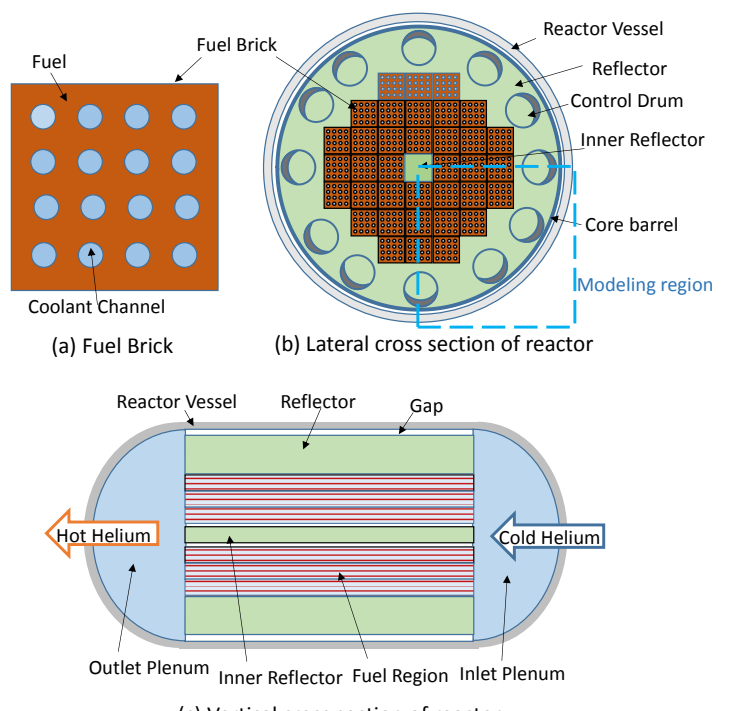

(c) Vertical cross section of reactor

Figure 2. Sketch of reactor structure.

\section{Modelica model}

The Modelica model of the micro gas-cooled reactor system was established, as shown in Figure 3. The model consists of five subsystems and the data exchange is accomplished by Modelica interface. The five subsystems are described as follows:

(1) Reactor system. The core heat generated by the reactor system provides the energy source for the Brayton cycle. The reactor system focuses on the core reactivity, nuclear power, thermal-hydraulic, decay heat power and residual heat removal power. The system includes the models of the point reactor, decay heat power and thermal-hydraulic.

(2) Heat engine system. The heat engine system focuses on the Brayton cycle to realize the heat-work conversion. The system includes the models of turbine, compressor, regenerator, precooler, intercooler, and pipeline.

(3) Heat sink system. The heat sink system provides the cold source for the precooler and intercooler in the heat engine system, and is simply realized by mass inlet boundary.

(4) Electricity system. The electricity system converts the rotational kinetic energy in the heat engine system into the electricity energy, and is simply realized by a given load.

(5) Control system. The control system focuses on the control of the power operation and heat sink.

The models of the reactor system, heat engine system and control system will be discussed detail below.

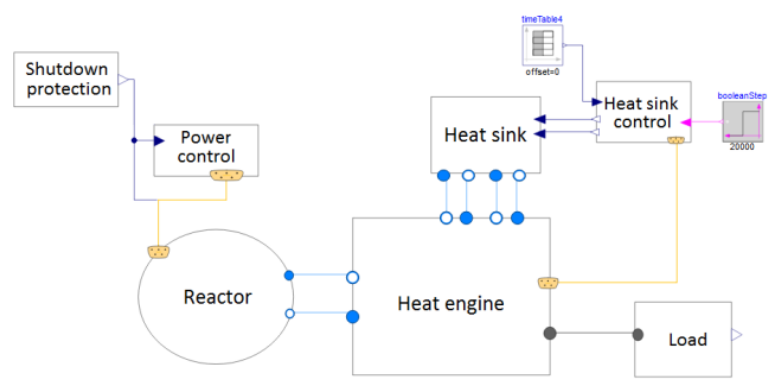

Figure 3. Modelica model of the micro gas-cooled reactor system.

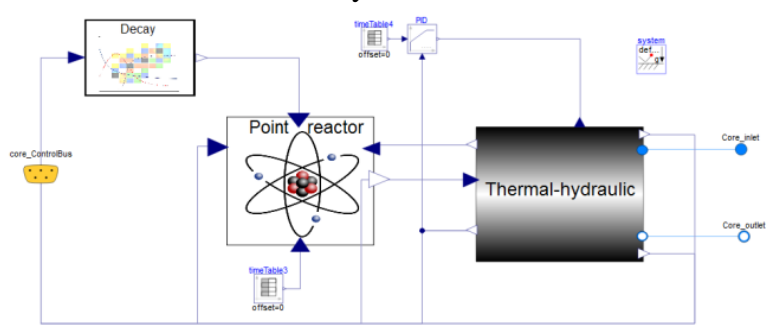

Figure 4. Modelica model of the reactor model.

\subsection{Reactor system}

The Modelica model of the reactor system is shown in Figure 4, including the models of the point reactor, decay heat and thermal-hydraulic. The decay heat model is implemented by calling external $\mathrm{C}$ functions by virtue of the external function interface in MWorks. The helium inlet and outlet in the reactor system are connected to the helium outlet and inlet in the heat engine system, respectively.

\subsubsection{Point reactor model}

The reactor neutron model is based on the point reactor neutron dynamics. The three-dimensional effect of the neutrons space dynamics is neglected, and the neutron flux distribution is fixed in space and only change with time. The equations are listed as 
follows:

$$
\begin{gathered}
\frac{d N}{d t}=\frac{\rho-\beta}{\Lambda} N+\sum_{i=1}^{6} \lambda_{i} C_{i} \\
\frac{d C_{i}}{d t}=\frac{\beta_{i}}{\Lambda} N-\lambda_{i} C_{i} \\
\beta=\sum_{i=1}^{6} \beta_{i} \\
\rho=\rho_{i n i}+\rho_{T}+\rho_{\mathrm{Xe}}+\rho_{\text {ext }}
\end{gathered}
$$

where $N$ is the average neutron density, $\Lambda$ is the neutron generation time, $\beta$ is the total delayed neutron fraction, $\beta_{i}$ is the delayed neutron fraction of group $i, C_{i}$ is the precursor concentration of the delayed neutron of group $i, \lambda_{i}$ is the decay constant of the delayed neutron of group $i$, and $\rho$ is the net reactivity. The initial reactivity $\left(\rho_{\text {ini }}\right)$, temperature reactivity $\left(\rho_{T}\right)$, xenon reactivity $\left(\rho_{X e}\right)$ and external reactivity $\left(\rho_{\text {ext }}\right)$ introduced by control rods are considered in the model.

\subsubsection{Thermal-hydraulic model}

Thanks to the symmetry, one quarter of the whole reactor is chosen in the thermal-hydraulic (T-H) model as shown in Figure 5, including the fuel bricks, inlet and outlet plenums, and thermal components (reflectors, core barrel and pressure vessel). Since the major material of the control drums is same as the reflector, they are simply considered same and merged into the reflector component in the $\mathrm{T}-\mathrm{H}$ modeling.

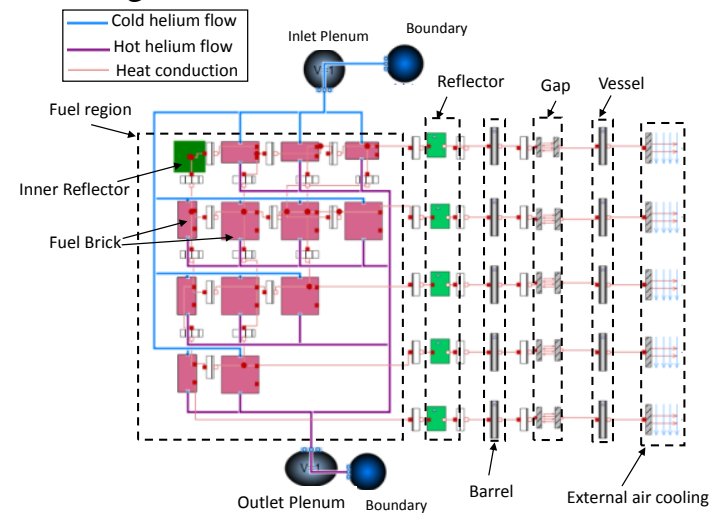

Figure 5. Modelica model of the reactor thermalhydraulic.

Since there is an amount of graphite inside core which is thermal conductor, the heat conduction phenomenon in the solid core regions is very important and need to be modeled. In reality, gaps may exist between the bricks, deteriorate the heat transfer. At this preliminary work, the assumption of no gap is taken and the heat conduction are ideally considered between the fuel bricks, reflectors and barrel.

Because the nuclear power in different fuel bricks are not uniform, the heat transfer phenomenon in the reactor region have the 3-dimentional behavior, which should be taken into account in the modeling and simulation. In this paper, a $\mathrm{T}-\mathrm{H}$ model with coarse 3-D nodalization is established by using the lumped parameter method.

The nodalization of the fuel region is shown in Figure 6. There are 13 lateral nodes each representing a brick, and the fuel bricks are divided into 5 segments axially. Therefore there are 65 blocks, of each contains a solid fuel block and a flow segment. Each solid fuel block has a heat source which receives the nuclear heat power, transfers heat energy to the neighbor solid fuel blocks vertically and laterally by thermal conduction, and releases heat energy into the gas segment inside it by convection.

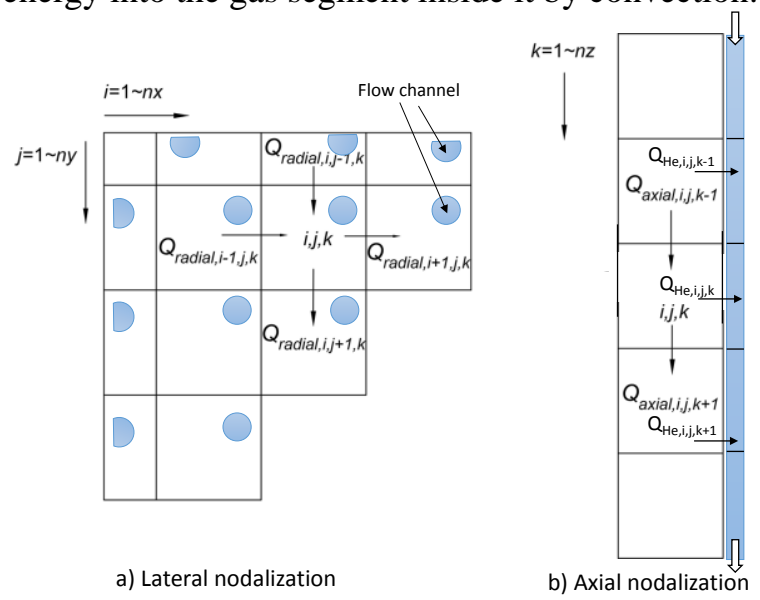

Figure 6. Nodalization of fuel region.

The energy function of the solid block is:

$$
\begin{aligned}
& \rho_{f, i, j, k} V_{f, i, j, k} C_{p, i, j, k} \frac{d T_{\text {block }, i, j, k}}{d t} \\
& =Q_{\text {nuclear }, i, j, k}+Q_{\text {radial }, i-1, j, k}+Q_{\text {radial }, i, j-1, k}+Q_{\text {axial }, i, j, k-1} \\
& -Q_{\mathrm{He}, i, j, k}-Q_{\text {radial }, i+1, j, k}-Q_{\text {radial }, i, j+1, k}-Q_{\text {axial }, i, j, k+1}
\end{aligned}
$$

where $i, j, k$ represent the coordinates, $V_{f}$ is the segment volume, $\rho_{f}$ is the density, $C_{p}$ is the specific heat capacity, $T_{\text {block }}$ is the temperature, $Q_{\text {nuclear }}$ is the input nuclear energy, $Q_{\mathrm{He}}$ is the output heat convection energy with helium, $Q_{\text {radial }}$ is the heat conduction energy with the radial segments of the surrounding fuel assembly, $Q_{\text {axial }}$ is the heat conduction energy with the axial segments within the fuel assembly.

Since no cross flow between flow channels, a flow channel can be modeled as a pipe using the Modelica standard library. The helium compression is considered and the heat convection is calculated as (Hale et al. 2015):

$$
\begin{gathered}
Q_{\mathrm{He}}=h_{\mathrm{He}} A_{\mathrm{He}}\left(T_{\text {block }}-T_{\mathrm{He}}\right) \\
h_{\mathrm{He}}=\frac{\lambda_{\mathrm{He}}}{D_{\text {channel }}} \mathrm{Nu}_{\mathrm{He}} \\
\mathrm{Nu}_{\mathrm{He}}= \begin{cases}2\left(\frac{m_{\mathrm{He}} C_{p, \mathrm{He}}}{\lambda_{\mathrm{He}} l}\right)^{0.333} & \operatorname{Re}_{\mathrm{He}}<\operatorname{Re}_{\text {crtical }} \\
0.023 \mathrm{Re}_{\mathrm{He}}^{0.8} \operatorname{Pr}_{\mathrm{He}}^{0.4} & \operatorname{Re}_{\mathrm{He}} \geq \operatorname{Re}_{\text {crtical }}\end{cases}
\end{gathered}
$$


where $Q_{\mathrm{He}}$ is the heat convection energy, $h_{\mathrm{He}}$ is the heat convection coefficient, $A_{\mathrm{He}}$ is the heat transfer area, $T_{\mathrm{He}}$ is the helium temperature, $\lambda_{\mathrm{He}}$ is the conductivity, $c_{p, \mathrm{He}}$ is the specific heat capacity, $D_{\text {channel }}$ is the channel diameter, $m_{\mathrm{He}}$ is the helium mass flow rate, $l$ is the channel length, $\mathrm{Nu}_{\mathrm{He}}$ is Nusselt number, $\mathrm{Pr}_{\mathrm{He}}$ is Prandtl number, $\mathrm{Re}_{\mathrm{He}}$ is Reynolds number, $\mathrm{Re}_{\text {critical }}$ is the critical Reynolds number between the laminar and turbulent.

The T-H modeling of the reflector and vessel are similar and simpler to the modeling of fuel region, also related to the heat conduction.

For the gap between the barrel and vessel, heat radiation is assumed to be the only heat transfer process. For the external vessel cooling, the air convection is simply modeled by setting a fixed convection heat transfer coefficient.

\subsection{Heat engine system}

As shown in Figure 7, the heat engine system adopts direct Brayton cycle to accomplish the heat-work conversion, and the system is composed of the turbine, compressors, recuperator, precooler, intercoolor, and pipes. The high pressure helium flows through the reactor core and gets heated, and then flows into the turbine to expand and does work to drive the main shaft to rotate. The rotating main shaft drives the compressors to compress the helium at the same time. The helium with relatively high temperature from the turbine goes through the lower pressure side of the recuperator and transfer heat to the high pressure helium which comes from the high pressure compressor. After that, the helium goes into the precooler to reduce the temperature and then enters the low pressure compressor. Then, the helium with low temperature and low pressure is compressed by the low and high compressors, which are connected by the intercooler, and thus the helium pressure is increased. Then the helium goes through the higher pressure side of the recuperator, and the temperature rises approximately to that at the turbine outlet. Finally, the helium flows into the reactor core and repeats such thermal cycle process again. The turbine model and compressor model is established to simulate the turbine and compressor. The heat exchanger model is established to simulate the recuperator, precooler, and intercooler. The working medium in the two sides of the precooler and intercooler are helium and cooling water, while the working medium in the two sides of the recuperator are both helium. The helium inlet and outlet in the heat engine system are connected to the helium outlet and inlet in the reactor system, respectively.

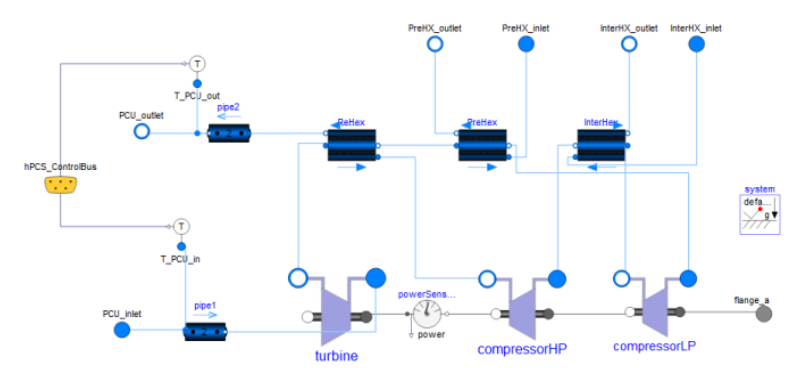

Figure 7. Modelica model of the heat engine system.

It is supposed that the helium flow is stable in the turbine and compressor, and the process is adiabatic. The ThermoPower library is used to establish the model of turbine and compressor. The characteristic curves, which are commonly used in the practical engineering, are used to describe the working process (Fernández-VillacéandPaniagua 2010).

The heat exchanger model is based on the models of pipe, heat components, heat conduction, interface and medium physical properties, as shown in Figure 8 . The model of heat transfer and pressure drop in the pipe model are used to simulate the heat transfer and flow resistance between the fluid and pipe wall, and the heat conduction model is used to simulate the heat conduction between the cold and hot channel. The heat exchanger is divided into several segments, which are combined by the heat conduction model.

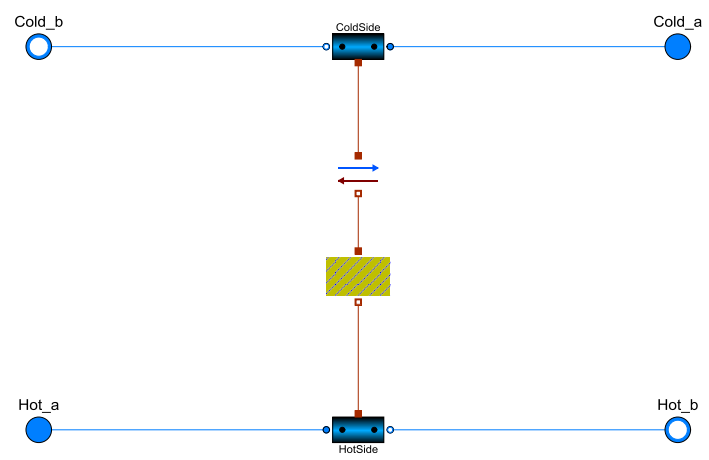

Figure 8. Modelica model of the heat exchanger.

\subsection{Control system}

The control system includes the power operation control and heat sink control in current research stage, as shown in Figure 9 and 10, respectively. The former controls the motion of control rods in the reactor system according to the monitored power and reactivity. The latter controls the helium flow rate in the precooler and intercooler in the heat sink system according to the helium temperature. The control system can be used to analyze the coupling matching of the reactor system and heat engine system, and it can also be used to explore the operation modes. 


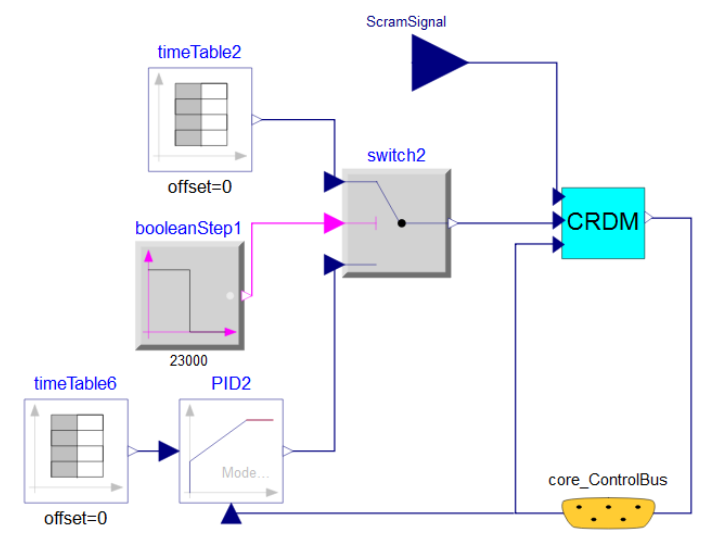

Figure 9. Modelica model of power operation control system.

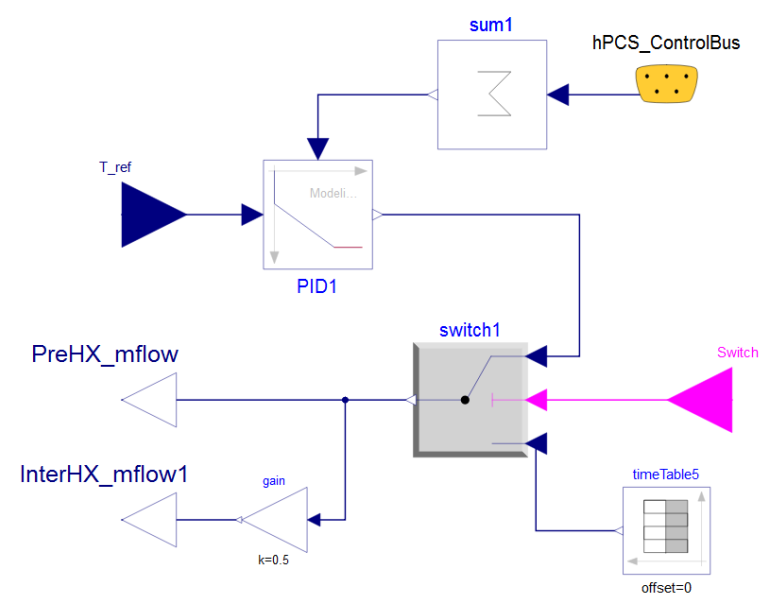

Figure 10. Modelica model of heat sink control system.

\section{Calculation and analysis}

Based on the above-mentioned theories and Modelica models, two simulations of the micro gas-cooled reactor design were carried out. One is the extreme accident scenario and the other is a normal reactorengine load-following operation.

\subsection{Extreme accident}

The extreme accident is defined as such scenario, where the heat engines shut down and the helium flow rate drops to zero, with all of the control rods withdrew from the core. When the accident happens, the heat from the reactor core will transfer to the outer wall of the reactor pressure vessel, and then removed by passive air cooling in the environment. It is supposed that the accident happens after the reactor works at full power for $80 \mathrm{~h}$. The various powers and average core temperature are shown in Figure 11 through Figure 13.

The control rods withdraw from core, introducing positive reactivity to the reactor core, and then the fission power rapidly rise up to 1.8 times of the full power in 80s. As a result, the core temperature rises promptly, leading to the rapidly increased negative temperature reactivity, and consequently the total reactivity decreases to a negative value. Therefore, the fission power drops to nearly zero, and the reactor shuts down. However, the total reactor thermal power is not zero because of the decay heat result from the continually generated fission products and activation products. With the residual heat removal, the reactor core temperature gradually goes down, leading to the decrease of negative temperature reactivity. Then the net reactivity increases and becomes positive after about $1.2 \mathrm{~h}$ of the accident, and thus the reactor achieves re-criticality. The final re-criticality power is about $5.2 \%$ of the full power, and the total thermal power which is the summation of the fission power and the decay heat power is equal to the residual heat removal power through the pressure vessel wall.

The maximum fuel temperature after the accident is about $1211{ }^{\circ} \mathrm{C}$ (The fuel and graphite is not separated, and the real maximum fuel temperature will be higher than the simulated value), which is lower than the design limit temperature $\left(1600^{\circ} \mathrm{C}\right)$. Therefore, the reactor shutdown can be achieved after the accident only by the negative reactivity result from the increase of core temperature, and the reactor has good inherent safety. Tsinghua University has done the similar accident experiments on high temperature gas-cooled reactor-test module (HTR-10) reactor in 2003 (Gou et al. 2018), and the reactor shutdown was realized automatically by the negative reactivity result from the increase of core temperature. The safety of fuel and core can be guaranteed.

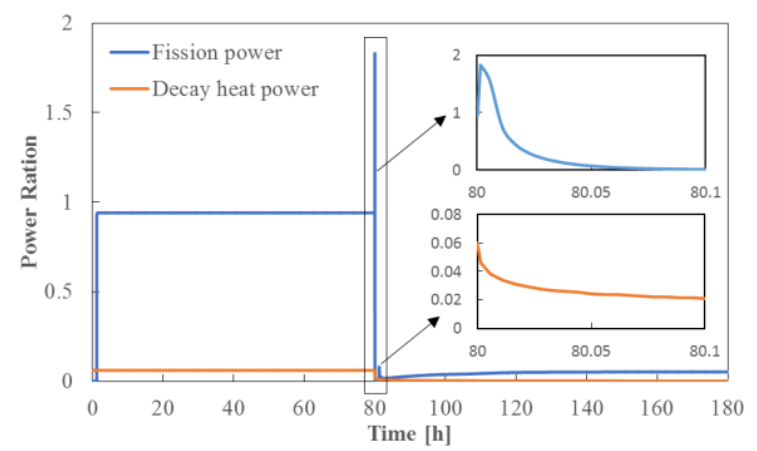

Figure 11. Nuclear fission power and decay heat power.

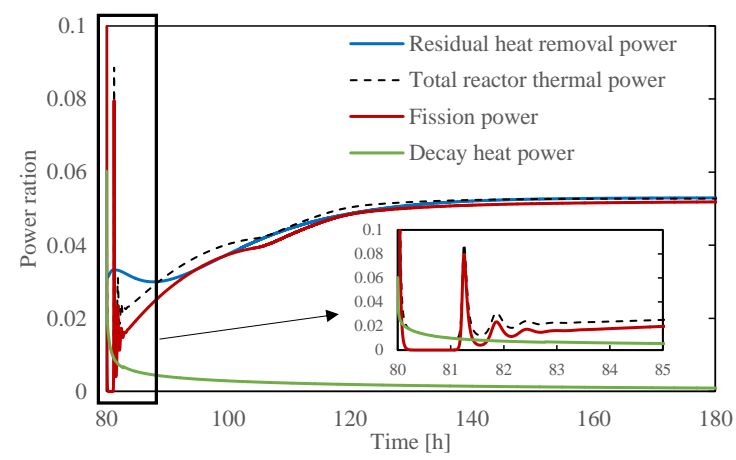

Figure 12. Reactor power and residual heat removal power. 


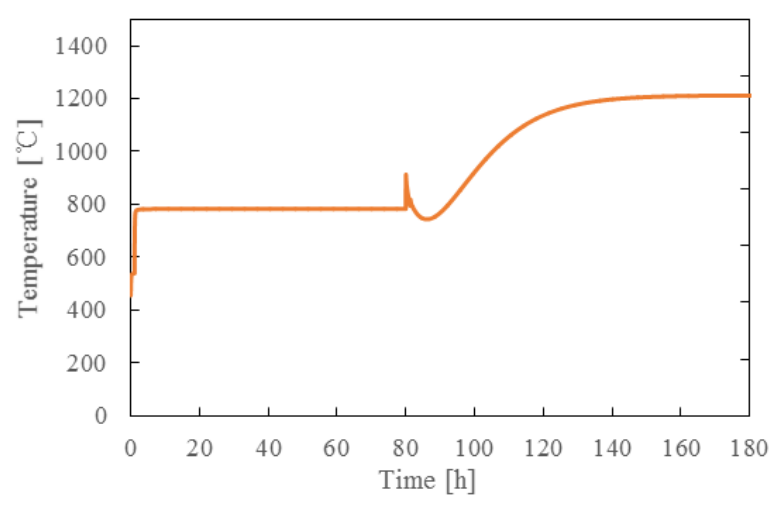

Figure 13. Core average temperature.

\subsection{Reactor-engine load-following}

It is supposed that the actual electricity load is timedependent and fluctuates periodically. The fluctuation period is $24 \mathrm{~h}$, which consists of peak period, steady period and low period. The demand power is determined by the electricity load, as shown in Figure 15. By simply adjusting the helium inventory (or pressure, setup in MWorks as shown in Figure 14) and the control rod position, the reactor nuclear power and net output power change periodically and synchronously, and the net output power coincides well with the demand power, which demonstrates that the function of load-following is realized successfully.

The reactor nuclear power and net output power are $4.10 \mathrm{MW}$ and $1.37 \mathrm{MW}$ respectively, during the peak period, and are 2.10 MW and 0.69 MW respectively, during the low period. The electric power generation efficiency is defined as the ratio of the net output power to the nuclear power, which changes little and nearly $33.0 \%$, as shown in Figure 16.

As shown in Figure 17, the helium temperature at the core inlet and outlet are about $435^{\circ} \mathrm{C}$ and $750^{\circ} \mathrm{C}$, respectively, and both change periodically with a light fluctuation $\left( \pm 3^{\circ} \mathrm{C}\right)$.

As shown in Figure 18, the negative temperature reactivity changes little because of the nearly constant core temperature. The negative xenon reactivity changes periodically because of the periodically changed xenon concentration, which result from the fluctuating reactor power. Therefore, the external reactivity introduced by control rods also changes periodically to keep the reactor critical.

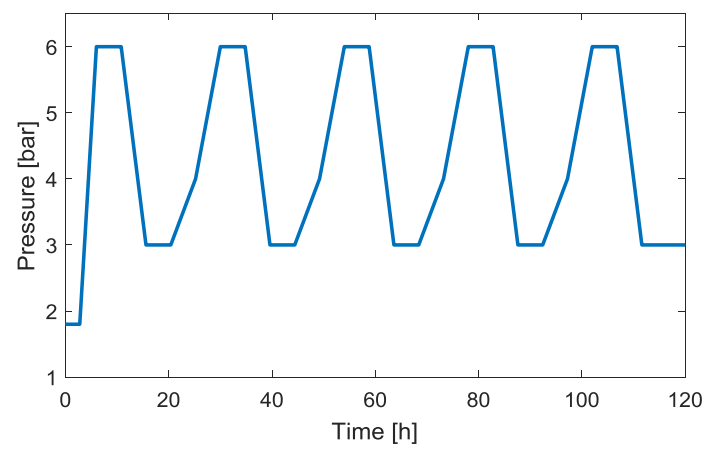

Figure 14. Helium pressure.

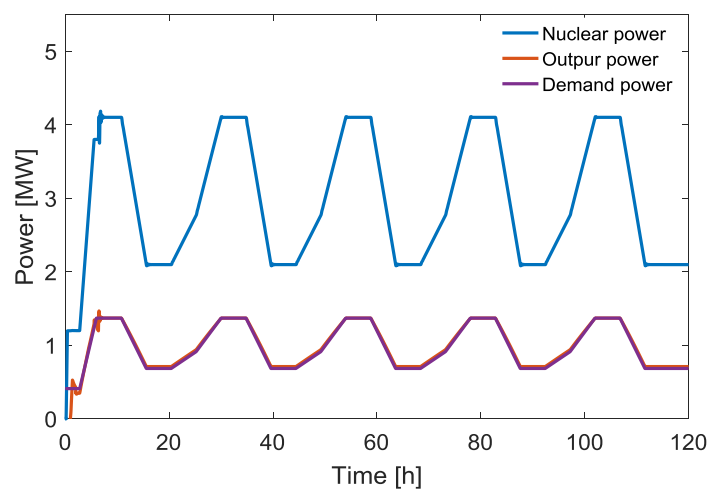

Figure 15. Nuclear power and output power.

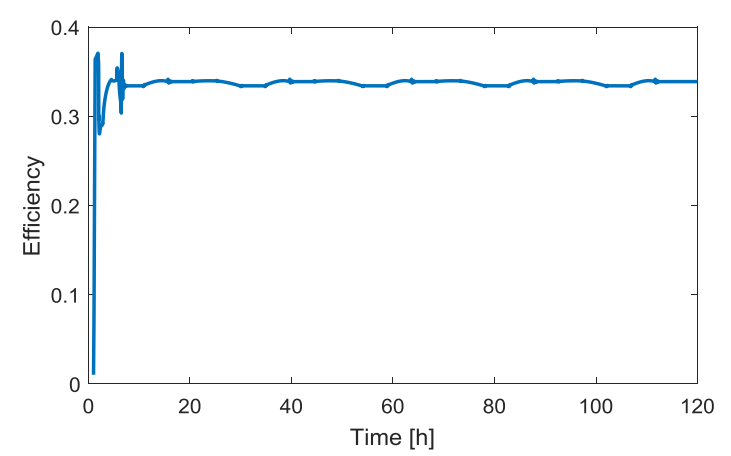

Figure 16. Power generation efficiency.

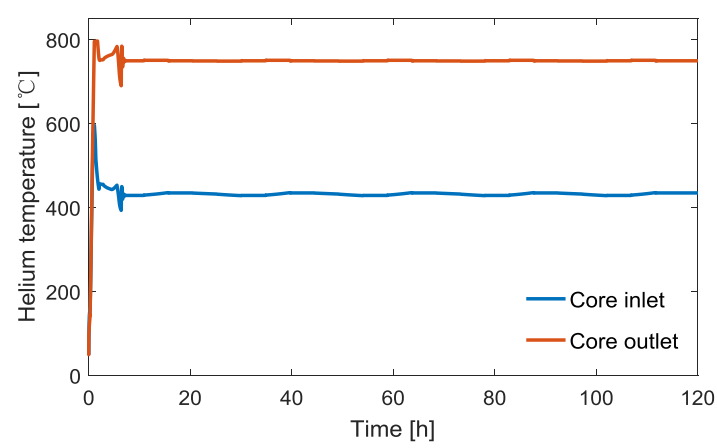

Figure 17. Helium temperature at core inlet and outlet. 


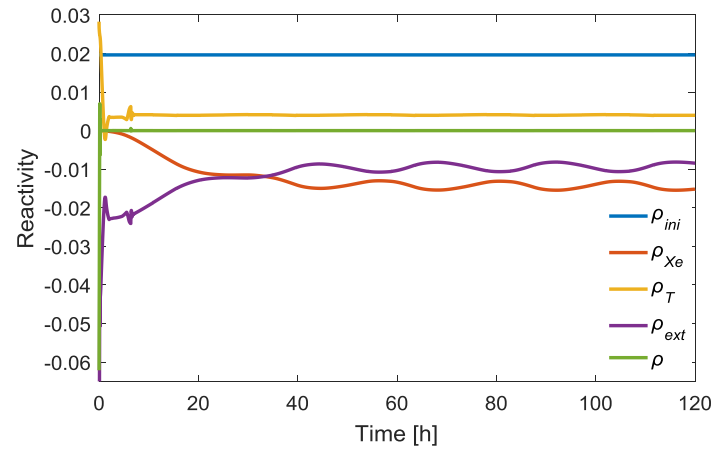

Figure 18. Core reactivity.

\section{Conclusions}

The system modeling and simulation for a micro gascooled reactor were carried out by Modelica language. The extreme accident and load-following were taken as examples to calculate and analyze. The simulation results showed that the reactor has good inherent safety even under the extreme accident, in which the reactor shutdown can be achieved only by the negative reactivity result from the increase of core temperature and the fuels were not damaged since the decay heat was removed by passive air cooling from outside of the reactor pressure vessel. The reactor also has good load-following performance, which can be achieved by simply adjusting the helium inventory (or pressure) and the control rod position, while the core temperature and power generation efficiency kept constant.

It should be mentioned that this Modelica model focus the system dynamic behavior, in which many simplifications have been taken. More verification will be carried out in future by comparing with the simulation results of high fidelity simulations or experiments. The simulation results is reasonable and accepted for the system dynamic analysis.

\section{References}

Bainan, Z., Faren, Q., Tao, X., Yang, L., \& Wei, W. (2020). Model based development method of manned spacecraft: Research and practice[J]. Acta Aeronauticaet Astronautica
Sinica, $\quad$ 41(7), 23967-023967.

DOI:

https://doi.org/10.7527/s1000-6893.2020.23967

Fernández-Villacé, V., \& Paniagua, G. (2010). "Simulation of a combined cycle for high speed propulsion". Paper presented at the 48th AIAA Aerospace Sciences Meeting Including the New Horizons Forum and Aerospace Exposition. DOI: https://doi.org/10.2514/6.2010-1125

Gou, F., Liu, Y., Chen, F.-B., \& Dong, Y.-J. (2018). Thermal behavior of the HTR-10 under combined PLOFC and ATWS condition initiated by unscrammed control rod withdrawal. Nuclear Science and Techniques, 29(9), 1-9. DOI: https://doi.org/10.1007/s41365-018-0472-3

Greenwood, M. S. (2018). "Molten Salt-Fueled Nuclear Reactor Model for Licensing and Safeguards Investigations" (1650-3686). URL: https://www.osti.gov/servlets/purl/1509585

Greenwood, M. S., Betzler, B. R., \& Qualls, A. L. (2018). "Dynamic System Models for Informing Licensing and Safeguards Investigations of Molten Salt Reactors". URL: https://www.osti.gov/servlets/purl/1456790.

Hale, R., Fugate, D., Cetiner, M., Ball, S., Qualls, A., \& Batteh, J. (2015). Update on ORNL TRANSFORM Tool: Preliminary Architecture/Modules for High-Temperature Gas-Cooled Reactor Concepts and Update on ALMR Control. ORNL/SPR-2015/367, Oak Ridge National Laboratory.

URL: https://www.ornl.gov/publication/update-ornl-transformtool-preliminary-architecture-modules-high-temperaturegas- 0

Rader, J. D., Smith, M. B., Greenwood, M. S., \& Harrison, T. (2019). "Nuclear Thermal Propulsion Dynamic Modeling with Modelica". URL: https://www.osti.gov/servlets/purl/1543223.

Yang, L., Liu, B., Shao, Y., Liang, T., \& Tang, C. (2010). The failure mechanisms of HTR coated particle fuel and computer code. Chinese Journal of Nuclear Science and Engineering, 30(3), 210-215, 222. URL: https://inis.iaea.org/search/search.aspx?orig_q=RN:45021 255

Yanping, H., Xiaokang, Z., \& Ji, D. (2021). Simulation Model Architecture and Concept Validation for Thermal Hydraulic Characteristics of 9.59.Two-Phase Fluid Based on Modelica[J]. Nuclear Power Engineering, 42(1), 1. DOI: https://doi.org/10.13832/j.jnpe.2021.01.0001 